\title{
Newer non-vitamin K-antagonist direct oral anticoagulants in acute coronary syndromes
}

\author{
Andrea Rubboli \\ Division of Cardiology, Laboratory of Interventional Cardiology, Ospedale Maggiore, Bologna, Italy
}

\begin{abstract}
Standard dual antiplatelet therapy (DAPT) of aspirin and clopidogrel is associated with a substantial absolute incidence of adverse events, including death, myocardial infarction and stroke after an acute coronary syndrome (ACS). Combination therapy of an oral anticoagulant and DAPT has been previously proposed in order to improve efficacy, but has not gained popularity owing to the cumbersome management of vitamin K-antagonists (VKA). The recent introduction of newer, non-VKA, direct oral anticoagulants (NOAC), including dabigatran, apixaban, and rivaroxaban, has renewed the interest in combination therapy, owing to the more favorable pharmacokinetic and pharmacodynamic profiles of these drugs. Whereas phase II studies with dabigatran, apixaban, and rivaroxaban have consistently shown an increased bleeding risk with combination therapy, a potential increased efficacy has emerged for apixaban and rivaroxaban, thereby prompting phase III studies, namely APPRAISE-2 with apixaban and ATLAS ACS 2-TIMI 51 with rivaroxaban. Both APPRAISE-2 and ATLAS ACS 2-TIMI 51 studies confirmed a dose-dependent increase in major, including intracranial, bleeding with apixaban and rivaroxaban when combined with DAPT. Low-dose rivaroxaban on the other hand, was associated with significantly higher efficacy on the occurrence of combined cardiovascular death, myocardial infarction, or stroke, as well as of cardiovascular death, myocardial infarction and stent thrombosis. Owing to the persistent uncertainty regarding the net clinical benefit of combined therapy of NOAC, namely low-dose rivaroxaban, and DAPT, further studies are warranted to identify the ACS patient who will benefit most from such treatment, also in comparison to current standard DAPT of aspirin and prasugrel or ticagrelor.
\end{abstract}

\section{Introduction}

Dual antiplatelet therapy (DAPT) of aspirin and clopidogrel has long been the standard antithrombotic therapy following an acute coronary syndrome (ACS) ${ }^{1,2}$ While being significantly more effective than aspirin alone, ${ }^{3}$ such treatment is associated with a substantial absolute incidence of major adverse events, including death, myocardial infarction, or

Correspondence: Andrea Rubboli, Division of Cardiology, Laboratory of Interventional Cardiology, Ospedale Maggiore, Largo Nigrisoli 2, 40133 Bologna, Italy.

Tel.: +39.051.6478976 - Fax: +39.051.6478635.

E-mail: andrearubboli@libero.it

Key words: acute coronary syndrome, non-vitamin K-antagonist direct oral coagulants, rivaroxaban, low-dose, dual antiplatelet therapy.

Received for publication: 11 March 2013.

Revision received: Not required.

Accepted for publication: 9 April 2013

This work is licensed under a Creative Commons Attribution NonCommercial 3.0 License (CC BY-NC 3.0).

(C) Copyright A. Rubboli, 2013

Licensee PAGEPress, Italy

Italian Journal of Medicine 2013; 7(s8):22-28

doi:10.4081/itjm.2013.s8.22 stroke at 12-month follow up. For example, in the aspirin and clopidogrel arm of the Platelet Inhibition and Patient Outcomes (PLATO) study ${ }^{4}$ including patients with either non-ST-elevation and ST-elevation ACS who were treated according to best contemporary practice (i.e. aspirin in $94 \%$ of cases, clopidogrel in $100 \%$, beta-blockers in $89 \%$, statins in $89 \%$, parenteral anticoagulation in $100 \%$, glycoprotein IIb/IIIa inhibitors in $26 \%$, and percutaneous coronary intervention in $61 \%$ ), the 12 -month incidence of death, myocardial infarction and stroke was $12.3 \%, 5.9 \%, 6.9 \%$, and $1.3 \%$, respectively.

Whereas such a finding may be, at least partially, attributed to the persistent high on-treatment platelet reactivity (so-called resistance) which has been shown to affect up to $30 \%$ of patients treated with clopidogrel, therefore impairing its antiplatelet effect, ${ }^{5}$ the persistent activation of coagulation mechanisms which has been reported for $\mathrm{ACS}^{6}$ may also play a role. Indeed, the combination of aspirin and the oral anticoagulation warfarin after ACS has been shown to have a greater effect than aspirin alone on the occurrence of myocardial infarction and stroke. ${ }^{7}$ This comes, however, at the cost of an approximately 2.5 -fold higher incidence of major bleeding. ${ }^{7}$ Because of this, and of the several limitations of warfarin therapy (e.g. non-linear dose-response relationship, drug and food interactions, need for routine laboratory monitoring), the combination of aspirin and warfarin as antithrom- 
botic therapy after an ACS has never gained much popularity.

The recent development of newer, non-vitamin Kantagonists, direct oral anticoagulants (NOAC), including the thrombin inhibitor dabigatran and the factor $\mathrm{Xa}$ inhibitors apixaban and rivaroxaban, which show more favorable pharmacokinetic and pharmacodynamic profiles allowing for fixed-dose administration with no need for routine laboratory monitoring, have renewed the interest in the combination of aspirin and oral anticoagulants as antithrombotic therapy after an ACS.

\section{Phase II trials}

In the Dose Finding Study for Dabigatran Etexilate in Patients with Acute Coronary syndrome (RE-DEEM) trial, ${ }^{8} 1861$ patients with a recent myocardial infarction receiving DAPT with aspirin and clopidogrel were randomized to placebo or dabigatran at the doses of 50,75, 110 , and $150 \mathrm{mg}$ twice daily (Table 1 ). After six months of treatment, there was a dose-dependent 2- to 4-fold increase in major or clinically relevant non-major bleeding, as well as a significant reduction in coagulation activity as measured by D-dimer concentration. Evidence of efficacy of dabigatran was less evident, but the study was not powered to determine a difference in the incidence of ischemic events.

In the Apixaban for Prevention of Acute Ischemic Safety Events (APPRAISE) trial, ${ }^{9} 1715$ patients with a recent ACS were randomized to six months of one of the following apixaban doses: $2.5 \mathrm{mg}$ twice daily, $10 \mathrm{mg}$ once daily, $10 \mathrm{mg}$ twice daily or $20 \mathrm{mg}$ once daily, or placebo (Table 1). Nearly all patients received aspirin and $76 \%$ received clopidogrel. The primary outcome was major or clinically relevant non-major bleeding, and the secondary end point was cardiovascular death, myocardial infarction, severe recurrent is- chemia or ischemic stroke. The two higher-dose arms of apixaban were discontinued early due to increased bleeding events. In the remaining treatment arms, a dose-dependent increase in bleeding in addition to a trend toward fewer ischemic events was found at six months with apixaban compared to placebo. Safety and efficacy outcomes varied depending on the antiplatelet regimen: in those taking DAPT, the risk of bleeding was more pronounced and the reduction of ischemic events was less apparent than in those taking only aspirin.

The Anti-Xa Therapy to Lower Cardiovascular Events in Addition to Standard Therapy in Subjects with Acute Coronary Syndrome-Thrombolysis in Myocardial Infarction 46 trial $^{10}$ enrolled 3491 patients with a recent ACS and assigned them to six months of one of the following daily doses of rivaroxaban: $5 \mathrm{mg}$, $10 \mathrm{mg}, 15 \mathrm{mg}, 20 \mathrm{mg}$ or placebo (Table 1). These total daily doses were given either once daily or divided into twice-daily regimens. Furthermore, patients were divided into two groups on the basis of planned single antiplatelet therapy or DAPT. Approximately $78 \%$ of patients received DAPT, whereas the remaining received aspirin alone. The primary safety outcome was clinically significant major bleeding, minor bleeding or bleeding requiring medical attention. The primary efficacy end point was a composite of death, myocardial infarction stroke or severe recurrent ischemia requiring revascularization. At six months, rivaroxaban resulted in a dose-dependent increased risk of clinically significant bleeding both in patients receiving aspirin alone and, even more so, in patients receiving DAPT. In addition, both patients receiving once and twice-daily dosing had a dose-dependent increased risk of bleeding. Compared with placebo, rivaroxaban was associated with a trend toward fewer ischemic outcomes, as well as a nominally significant reduction in death, myocardial infarction and stroke.

Table 1. Phase II clinical trials with NOAC.

\begin{tabular}{lccc}
\hline & Dabigatran etexilate & Apixaban & Rivaroxaban \\
\hline Acronym & RE-DEEM & ATLAS ACS-TIMI $46^{10}$ \\
\hline $\mathrm{n}$ & 1861 & APPRAISE ${ }^{9}$ & 3491 \\
\hline STEMI/NSTEMI, $\%$ & $60 / 40$ & $61-67 / 33-39$ & $52 / 48$ \\
\hline DAPT, $\%$ & 99 & 76 & 100 \\
\hline Duration of therapy, months & 6 & 6 & 6 \\
\hline Dosage & $50-150 \mathrm{mg}$ BID & $10-20 \mathrm{mg}$ OD/2.5-10 mg BID & $5-20 \mathrm{mg}$ OD \\
\hline Safety outcome, HR $(95 \% \mathrm{CI})$ & $50 \mathrm{mg}: 1.82(0.77-4.29)$ & $2.5 \mathrm{mg}$ BID: $1.78(0.91-3.48)$ & $5 \mathrm{mg}: 2.17(0.91-5.18)$ \\
& $75 \mathrm{mg}: 2.44(1.05-5.65)$ & $10 \mathrm{mg}$ BID: $2.45(1.31-4.61)$ & $10 \mathrm{mg}: 3.34(2.15-5.19)$ \\
& $110 \mathrm{mg}: 3.36(1.60-7.91)$ & $10 \mathrm{mg}$ BID and $20 \mathrm{mg}$ OD: & $15 \mathrm{mg}: 3.41(1.97-5.89)$ \\
& $150 \mathrm{mg}: 3.88(1.73-8.74)$ & Terminated prematurely because of bleeding & $20 \mathrm{mg}: 4.56(2.83-7.33)$
\end{tabular}

STEMI, ST-elevation myocardial infarction; NSTEMI, non-ST-elevation myocardial infarction; DAPT, dual antiplatelet therapy; HR, hazard ratio; CI, confidence intervals; BID, twice daily; OD, once daily. 
Owing to the promising results observed in the APPRAISE ${ }^{9}$ and ATLAS-ACS $46^{10}$ trials regarding the ischemic outcomes, suggesting that combination of apixaban or rivaroxaban with DAPT may have increased efficacy, phase III trials were designed.

\section{Phase III trials}

The Apixaban for Prevention of Acute Ischemic Events 2 (APPRAISE-2) trial ${ }^{11}$ is a double-blind, placebo-controlled trial comparing to placebo apixaban $5 \mathrm{mg}$ twice daily in addition to DAPT in patients with a recent acute coronary syndrome (i.e. myocardial infarction with ST-elevation or non-ST-elevation, or unstable angina). Eligible patients were also required to have two or more of the following high-risk characteristics: age 65 years or over, diabetes mellitus, myocardial infarction within previous five years, cerebrovascular disease, peripheral vascular disease, clinical heart failure or left ventricular ejection fraction more than $40 \%$ at the index event, impaired renal function (i.e. creatinine clearance $<60 \mathrm{~mL} / \mathrm{min}$ ) and no revascularization after the index event. The primary efficacy outcome was the composite of cardiovascular death, myocardial infarction, or ischemic stroke. The primary safety outcome was major bleeding, according to the Thrombolysis In Myocardial Infarction (TIMI) classification. ${ }^{12}$ The study was prematurely terminated at a median follow up of eight months due to an increase in bleeding events with apixaban without a meaningful reduction in ischemic events after 7392 patients were enrolled (approximately $70 \%$ of the planned population) (Table 2). Interestingly, also the incidences of intracranial (12 vs 3) and fatal (5 vs 0$)$ bleeding were higher with apixaban.

The Anti-Xa Therapy to Lower Cardiovascular Events in Addition to Standard Therapy in Subjects with Acute Coronary Syndrome-Thrombolysis In Myocardial Infarction (ATLAS ACS 2-TIMI 51) trial $^{13}$ is a double-blind, placebo-controlled trial comparing placebo with rivaroxaban 2.5 and $5 \mathrm{mg}$ twice daily in addition to DAPT in patients with a recent ACS (i.e. ST-elevation or non-ST-elevation myocardial infarction, or unstable angina). Patients were not required to have additional risk factors for recurrent ischemic events, and patients with previous gastrointestinal bleeding, intracranial hemorrhage or ischemic stroke or transient ischemic attack were excluded. The primary efficacy end point was a composite of cardiovascular death, myocardial infarction, or stroke. The primary safety end point was TIMI major bleeding not related to coronary artery bypass grafting. Testing was pre-specified to occur between the combined-dose (i.e. 2.5 and $5 \mathrm{mg}$ twice daily) group for rivaroxaban and placebo. If this comparison significantly favored rivaroxaban, then each of the two doses of rivaroxaban was simultaneously compared with placebo. A total of 15,526 patients were enrolled and followed-up for a mean of 13 months. Rivaroxaban significantly reduced the primary end point of cardiovascular death, myocardial infarction, or stroke, as a result of a significant reduction in cardiovascular death and myocardial infarction, but not of stroke (Table 3). In the analysis of the two doses of rivaroxaban, each of the doses reduced the primary efficacy end point (Table 4). The $2.5 \mathrm{mg}$ twice-daily dose of rivaroxaban reduced the risk of death from cardiovascular causes and the risk of death from any cause. The $5 \mathrm{mg}$ twice-daily dose reduced the risk of myocardial infarction. The risk of stent thrombosis was significantly reduced by combined-dose rivaroxaban as well as by each dose of the drug. Rivaroxaban significantly increased the rate of TIMI major bleeding, as well as of minor bleeding, bleeding requiring medical attention, and intracranial bleeding (Table 3). There was no significant difference in the rates of fatal bleeding associated with rivaroxaban compared to placebo (Table 3).

Table 2. Efficacy and safety outcomes in APPRAISE-2 trial. ${ }^{11}$

\begin{tabular}{|c|c|c|c|c|}
\hline & Apixaban (\%) & Placebo (\%) & HR (95\% CI) & $\mathbf{P}$ \\
\hline Primary efficacy end point (CV death, MI, ischemic stroke) & 7.5 & 7.9 & $0.95(0.80-1.11)$ & 0.51 \\
\hline $\mathrm{CV}$ death & 7.1 & 6.6 & $1.08(0.86-1.35)$ & 0.51 \\
\hline MI & 8.6 & 9.2 & $0.96(0.73-1.25)$ & 0.76 \\
\hline Ischemic stroke & 1.1 & 1.6 & $0.68(0.40-1.15)$ & 0.14 \\
\hline Stent thrombosis & 1.6 & 2.2 & $0.73(0.47-1.12)$ & 0.15 \\
\hline Primary safety end point (TIMI major bleeding) & 2.4 & 0.9 & $2.59(1.40-4.46)$ & 0.001 \\
\hline TIMI major or minor bleeding & 4.2 & 1.5 & $2.79(1.83-4.27)$ & $<0.001$ \\
\hline Fatal bleeding & 0.3 & 0 & NA & NA \\
\hline Intracranial bleeding & 0.6 & 0.2 & $4.06(1.15-14.38)$ & 0.03 \\
\hline Net clinical outcome (CV death, MI, stroke, fatal bleeding) & 14.0 & 14.3 & $0.98(0.83-1.15)$ & 0.80 \\
\hline
\end{tabular}

CI, confidence intervals; CV, cardiovascular; MI, myocardial infarction; NA, not available. 


\section{Critical considerations}

Similar to phase II trials, ${ }^{8-10}$ phase III trials APPRAISE-2 ${ }^{11}$ and ATLAS ACS 2-TIMI $51^{13}$ have both demonstrated a dose-dependent increase in bleeding with the addition of an anticoagulant (i.e. factor Xa inhibitors apixaban or rivaroxaban) to DAPT. Only rivaroxaban in the ATLAS ACS 2-TIMI 51 trial $^{13}$ was found to have a benefit on ischemic events, and perhaps a greater benefit at the lower dose. APPRAISE$2^{11}$ was stopped early for safety concerns. Some uncertainties, therefore, remain regarding the efficacy of apixaban. Whereas both the APPRAISE- $2^{11}$ and the ATLAS ACS 2-TIMI $51^{13}$ trials studied direct factor Xa inhibitors in patients with recent ACS, two major differences between the studies are apparent, thereby possibly explaining the lack of consistency in efficacy outcomes (Table 5).

The patient population in APPRAISE- 2 trial ${ }^{11}$ was particularly high-risk for recurrent ischemic events, as shown by the additional criteria (i.e. age $\geq 65$ years, diabetes mellitus, myocardial infarction within previous 5 years, cerebrovascular disease, peripheral vascular disease, clinical heart failure or left ventricular ejection fraction $>40 \%$ at the index event, impaired renal function, and no revascularization after the index event) required to patients to be eligible for the study. This was not the case for the ATLAS ACS 2-TIMI 51 population, ${ }^{13}$ in which additional exclusion criteria were previous intracranial hemorrhage, previous ischemic stroke or transient ischemic attack. However, it is not obvious why this would have impacted the observed difference in efficacy since higher-risk patients typically have similar relative and thus greater absolute benefit from effective antithrombotic therapies. On the other hand, higher rates of bleeding may lead to subsequent thrombotic events, particularly if antithrombotic drugs are stopped in response to bleeding. There was no evidence of more benefit in the lower-risk patients in the ATLAS ACS 2-TIMI 51 trial. ${ }^{13}$ Ultimately, understanding the patient popula- tion who will obtain the most favorable risk/benefit profile from the addition of an anticoagulant to antiplatelet therapy after an ACS will require further investigation.

In addition to differences in patient populations, the APPRAISE-2 ${ }^{11}$ and ATLAS ACS 2-TIMI $51^{13}$ trials also investigated different anticoagulant doses. In the APPRAISE-2 trial, ${ }^{11}$ apixaban was given at the same dose of $5 \mathrm{mg}$ twice-daily used in atrial fibrillation, ${ }^{14}$ whereas in the ATLAS ACS 2-TIMI 51 trial, ${ }^{13}$ the doses were 2.5 or $5 \mathrm{mg}$ twice daily, which correspond to one-fourth and one-half, respectively, of the dose tested in atrial fibrillation. ${ }^{15}$ Whereas a lower dose of anticoagulant resulting in less bleeding makes sense, the observed increases in bleeding with apixaban in the APPRAISE- 2 trial ${ }^{11}$ and rivaroxaban in the ATLAS ACS 2-TIMI 51 tria $^{13}$ were similar. On the other hand, the observed better efficacy with a lower dose of an anticoagulant within the ATLAS ACS 2TIMI $51^{13}$ or between the ATLAS ACS 2-TIMI $51^{13}$ and APPRAISE- $2^{11}$ trials is counterintuitive. Lower doses of an oral anticoagulant may have a more favorable risk/benefit profile, but full understanding of the ideal dosing regimen for these agents will require further study.

Interestingly, in the ATLAS ACS 2-TIMI 51 trial, ${ }^{13}$ rivaroxaban at any dose was highly effective in preventing stent thrombosis, therefore making a point for combined treatment of rivaroxaban, aspirin, and clopidogrel in patients at risk for such a rare but highly unfavorable complication.

As regards the course of major and extracranial bleedings in the APPRAISE-2 ${ }^{11}$ and ATLAS ACS 2TIMI $51^{13}$ trials, it is interesting to note that they occurred throughout the whole treatment period in both trials. The gain in ischemic events in the ATLAS ACS 2-TIMI 51 study ${ }^{13}$ was, on the other hand, greater later rather than earlier during follow up, therefore reducing the value of opting for short-term anticoagulant treatment to avoid the accrual of major bleeding events in the long term. Therefore, the unavoidable excess in

Table 3. Efficacy and safety outcomes in ATLAS ACS 2-TIMI 51 trial. $^{12}$

\begin{tabular}{|c|c|c|c|c|}
\hline & Rivaroxaban (\%) & Placebo (\%) & HR (95\% CI) & $\mathbf{P}$ \\
\hline Primary efficacy outcome (CV death, MI, stroke) & 8.9 & 10.7 & $0.84(0.74-0.96)$ & 0.002 \\
\hline CV death & 3.3 & 4.1 & $0.80(0.65-0.99)$ & 0.05 \\
\hline MI & 5.5 & 6.6 & $0.85(0.72-1.00)$ & 0.01 \\
\hline Stroke & 1.6 & 1.2 & $1.24(0.86-1.78)$ & 0.19 \\
\hline Stent thrombosis & 2.3 & 2.9 & $0.69(0.61-0.93)$ & 0.008 \\
\hline Primary safety outcome (TIMI major bleeding non-CABG-related) & 2.1 & 0.6 & $3.96(2.46-6.38)$ & $<0.001$ \\
\hline TIMI minor bleeding & 1.3 & 0.5 & $2.07(1.27-3.37)$ & 0.003 \\
\hline TIMI bleeding requiring medical attention & 14.5 & 7.5 & $2.09(1.83-2.38)$ & $<0.001$ \\
\hline Intracranial bleeding & 0.6 & 0.2 & $3.28(1.28-8.42)$ & 0.00 \\
\hline Fatal bleeding & 0.3 & 0.2 & $1.19(0.54-2.59)$ & 0.66 \\
\hline
\end{tabular}

$\mathrm{CV}$, cardiovascular; $\mathrm{MI}$, myocardial infarction; $\mathrm{CABG}$, coronary-artery bypass grafting. 
major and extracranial bleeding is a major concern when the combination of oral inhibitors of factor Xa such as apixaban and rivaroxaban with DAPT is considered in patients with an ACS.

A further consideration is that clopidogrel, which has been used for DAPT both in the APPRAISE-2 ${ }^{11}$ and in the ATLAS ACS 2-TIMI $51^{13}$ trials, is no longer recommended as the $\mathrm{P} 2 \mathrm{Y}_{12}$-receptor inhibitor to be associated with aspirin after an ACS. Based on the results of the Trial to Assess Improvement in Therapeutic Outcomes by Optimizing Platelet Inhibition with Prasugrel-Thrombolysis In Myocardial Infarction 38 (TRITON-TIMI) $38^{16}$ and PLATO $^{4}$ trials, in which the addition to aspirin of the newer $\mathrm{P}_{2} \mathrm{Y}_{12}$-receptor inhibitors prasugrel and ticagrelor, respectively, in patients with an ACS proved significantly more effective than clopidogrel, the most recent European Society of Cardiology guidelines for the treatment of both ST-elevation ${ }^{17}$ and non-ST-elevation $\mathrm{ACS}^{18}$ recommend that either one of these newer antiplatelet agents should be added to aspirin, and clopidogrel should be reserved only for those patients who for some reason cannot take prasugrel or ticagrelor. The question that arises is, therefore, whether combined therapy with rivaroxaban $2.5 \mathrm{mg}$ twice daily, aspirin, and clopidogrel may be preferable to the more convenient DAPT with aspirin and a newer $\mathrm{P}_{2} \mathrm{Y}_{12}$-receptor inhibitor. While acknowledging that indirect comparisons are not methodologically accurate, it is apparent that there are many similarities between the patient population enrolled in the $\mathrm{PLATO}^{4}$ trial and in the ATLAS ACS 2TIMI $51^{13}$ trial: mean age was 62 years in both,

Table 4. Efficacy and safety outcomes in ATLAS ACS 2-TIMI 51 trial according to rivaroxaban dose. ${ }^{12}$

\begin{tabular}{|c|c|c|c|c|c|c|c|}
\hline & $\begin{array}{c}\text { Rivaroxaban } \\
2.5 \text { twice } \\
\text { daily }(\%)\end{array}$ & $\begin{array}{c}\text { Rivaroxaban } \\
5 \text { mg twic } \\
\text { e daily (\%) }\end{array}$ & $\begin{array}{l}\text { Placebo } \\
\qquad \%)\end{array}$ & $\begin{array}{c}\text { HR (95\% CI) } \\
2.5 \text { mg twice } \\
\text { daily }\end{array}$ & $\mathbf{P}$ & $\begin{array}{c}\text { HR }(95 \% \text { CI }) \\
5 \text { mg twice } \\
\text { daily }\end{array}$ & $\mathbf{P}$ \\
\hline Primary efficacy outcome (CV death, MI, stroke) & 9.1 & 8.8 & 10.7 & $0.84(0.72-0.97)$ & 0.007 & $0.85(0.73-0.98)$ & 0.03 \\
\hline CV death & 2.7 & 4.0 & 4.1 & $0.66(0.51-0.86)$ & 0.005 & $0.94(0.75-1.20)$ & 0.63 \\
\hline MI & 6.1 & 4.9 & 6.6 & $0.90(0.75-1.09)$ & 0.09 & $0.79(0.65-0.97)$ & 0.02 \\
\hline Stroke & 1.4 & 1.7 & 1.2 & $1.13(0.74-1.73)$ & 0.56 & $1.34(0.90-2.02)$ & 0.15 \\
\hline Stent thrombosis & 2.2 & 2.3 & 2.9 & $0.65(0.45-0.94)$ & 0.02 & $0.73(0.51-1.04)$ & 0.04 \\
\hline $\begin{array}{l}\text { Primary safety outcome (TIMI major bleeding } \\
\text { non-CABG-related) }\end{array}$ & 1.8 & 2.4 & 0.6 & $3.46(2.08-5.77)$ & $<0.001$ & $4.47(2.71-7.36)$ & $<0.001$ \\
\hline TIMI minor bleeding & 0.9 & 1.6 & 0.5 & $1.62(0.92-2.82)$ & 0.09 & $2.52(1.50-4.24)$ & $<0.001$ \\
\hline TIMI bleeding requiring medical attention & 12.9 & 16.2 & 7.5 & $1.79(1.55-2.07)$ & $<0.001$ & $2.39(2.08-2.75)$ & $<0.001$ \\
\hline Intracranial bleeding & 0.4 & 0.7 & 0.2 & $2.83(1.02-7.86)$ & 0.04 & $3.74(1.39-10.07)$ & 0.005 \\
\hline Fatal bleeding & 0.1 & 0.4 & 0.2 & $0.67(0.24-1.89)$ & 0.45 & $1.72(0.75-3.92)$ & 0.20 \\
\hline
\end{tabular}

CI, confidence intervals; $\mathrm{CV}$, cardiovascular; $\mathrm{MI}$, myocardial infarction; $\mathrm{CABG}$, coronary-artery bypass grafting.

Table 5. Base-line characteristics, concomitant medication, and follow up.

\begin{tabular}{|c|c|c|}
\hline & APPRAISE-2 ${ }^{11}$ & ATLAS ACS 2-TIMI 51 ${ }^{12}$ \\
\hline $\mathrm{n}$ & 7392 & 15,526 \\
\hline Age, years & 67 (median) & 62 (mean) \\
\hline Diabetes mellitus, \% & 48 & 32 \\
\hline Heart failure, $\%$ & 40 & - \\
\hline Previous cerebrovascular disease & 10 & Excluded \\
\hline Renal function & $29 \%$ impaired & Median $\mathrm{CrCl} 85$ mL/min \\
\hline \multicolumn{3}{|l|}{ Index ACS, \% } \\
\hline STEMI & 40 & 50 \\
\hline NSTEMI & 42 & 26 \\
\hline Unstable angina & 18 & 24 \\
\hline Aspirin, \% & 97 & 99 \\
\hline Thienopyridine, $\%$ & 81 (DAPT) & 93 \\
\hline Follow up & 8 months (median) & 13 months \\
\hline
\end{tabular}

ACS, acute coronary syndrome; STEMI, ST-elevation myocardial infarction; NSTEMI, non-ST-elevation myocardial infarction; DAPT, dual antiplatelet therapy. 
Table 6. Outcome comparison between PLATO ${ }^{4}$ and ATLAS ACS 2-TIMI $51^{12}$ trials.

\begin{tabular}{lcc}
\hline & $\begin{array}{c}\text { Aspirin + ticagrelor } \\
\text { HR (95\% CI) }\end{array}$ & $\begin{array}{c}\text { Rivaroxaban 2.5 twice daily+aspirin+clopidogrel } \\
\text { HR (95\% CI) }\end{array}$ \\
\hline Primary end point (CV death, MI stroke) & $0.84(0.77-0.92) ; \mathrm{P}<0.001$ & $0.84(\mathrm{CI} 0.72-0.97) ; \mathrm{P}<0.007$ \\
\hline Death from CV causes & $0.88(0.81-0.95) ; \mathrm{P}<0.001$ & $0.66(0.51-0.86) ; \mathrm{P}=0.005$ \\
\hline Stent thrombosis & $0.77(0.62-0.95) ; \mathrm{P}=0.01$ & $0.65(0.45-0.94) ; \mathrm{P}=0.02$ \\
\hline Major bleeding & $1.03(0.93-1.15) ; \mathrm{P}=0.57$ & $3.46(2.08-5.77) ; \mathrm{P}<0.001$ \\
\hline Intracranial bleeding & $1.87(0.98-3.58) ; \mathrm{P}=0.06$ & $2.83(1.02-7.86) ; \mathrm{P}=0.04$ \\
\hline Fatal bleeding & $\mathrm{NA} ; \mathrm{P}=0.02$ & $0.67(0.24-1.89) ; \mathrm{P}=0.45$
\end{tabular}

CI, confidence intervals; CV, cardiovascular; MI, myocardial infarction; NA, not available.

percutaneous coronary intervention (PCI) was carried in $61 \%$ and $60 \%$ of cases, respectively, and indication for PCI was non-ST-elevation ACS in 60\% and 50\% of cases, respectively. When evaluating the efficacy and safety outcomes of the two trials, ${ }^{4,13}$ it appears that the benefit on the primary end point of cardiovascular death, myocardial, or stroke, as well as on cardiovascular death and stent thrombosis, was comparable between the more convenient DAPT of aspirin and ticagrelor, and the complex combination of rivaroxaban $2.5 \mathrm{mg}$ twice-daily, aspirin, and clopidogrel (Table 6). On the other hand, the incidence of total, as well as intracranial but not fatal bleeding appears to be substantially higher with the combination of rivaroxaban $2.5 \mathrm{mg}$ twice-daily, aspirin, and clopidogrel compared to DAPT of aspirin and ticagrelor (Table 6). Further investigation, therefore, appears to be needed to identify those ACS patients who may gain the most from a tailored therapy of oral anti-factor Xa anticoagulant (i.e. rivaroxaban), aspirin, and clopidogrel.

\section{Conclusions}

Newer, non-vitamin K-antagonists, direct oral anticoagulants, namely rivaroxaban at low dose, in association with DAPT represent a potentially beneficial treatment option for patients with a recent ACS. However, the contrasting efficacy results of the two phase III trials which have been conducted so far with the antifactor Xa apixaban and rivaroxaban (i.e. APPRAISE$2^{11}$ and ATLAS ACS 2-TIMI 51 ${ }^{13}$ ) show that the risks and benefits of these medications will need to be carefully weighed up and may depend on both patient risk profile and dosing of antithrombotic drugs. Whereas the increased risk of bleeding when these drugs are added to DAPT is unequivocal, the degree of efficacy and the relationship with patient risk and drug dose is less well established. A practical question is whether adding rivaroxaban to DAPT of aspirin and clopidogrel is more attractive than using DAPT of aspirin and ticagrelor, an approach also shown to prevent thrombotic events and reduce mortality, without a clear increase in major bleeding. Further investigation is warranted to accurately identify those patients who will benefit the most from the combined treatment with an oral anti-factor Xa anticoagulant (i.e. low-dose rivaroxaban) and DAPT of aspirin and clopidogrel.

\section{References}

1. Bertrand ME, Simoons ML, Fox KA, et al. Management of acute coronary syndromes: acute coronary syndromes without persistent ST segment elevation; recommendations of the Task Force of the European Society of Cardiology. Eur Heart J 2000;21:1406-32.

2. Van de Werf F, Bax J, Betriu A, et al. ESC Committee for Practice Guidelines (CPG). Management of acute myocardial infarction in patients presenting with persistent ST-segment elevation: the Task Force on the Management of ST-Segment Elevation Acute Myocardial Infarction of the European Society of Cardiology. Eur Heart J;29:2909-45.

3. The CURE Trial Investigators. Effects of clopidogrel in addition to aspirin in patients with acute coronary syndromes without ST-segment elevation. N Engl J Med 2001;345:494-502.

4. Wallentin L, Becker RC, Budaj A, et al. Ticagrelor versus clopidogrel in patients with acute coronary syndromes. N Engl J Med 2009;361:1045-57.

5. Sofi F, Marcucci R, Gori AM, et al. Clopidogrel non-responsiveness and risk of cardiovascular morbidity. An updated meta-analysis. Thromb Haemost 2010;103:841-8.

6. Merlini PA, Bauer KA, Oltrona L, et al. Persistent activation of coagulation mechanism in unstable angina and myocardial infarction. Circulation 1994;90:61-8.

7. Rothberg MB, Celestin C, Fiore LD, et al. Warfarin plus aspirin after myocardial infarction or the acute coronary syndrome: meta-analysis with estimates of risk and benefit. Ann Intern Med 2005;143:241-50.

8. Oldgren J, Budaj A, Granger CB, et al.; RE-DEEM Investigators. Dabigatran vs. placebo in patients with acute coronary syndromes on dual antiplatelet therapy: a randomized, double-blind, phase II trial. Eur Heart J 2011;32:2781-9.

9. Alexander JH, Becker RC, Bhatt DL, et al. Apixaban, 
an oral, direct, selective factor Xa inhibitor, in combination with antiplatelet therapy after acute coronary syndrome: results of the Apixaban for Prevention of Acute Ischemic and Safety Events (APPRAISE) trial. N Engl J Med 2009;119:2877-85.

10. Mega JL, Braunwald E, Mohanavelu S, et al. Rivaroxaban versus placebo in patients with acute coronary syndromes (ATLAS ACS TIMI 46): a randomized, doubleblind, phase II trial. Lancet 2009;374:29-38.

11. Alexander JH, Lopes R, James S, et al. Apixaban with antiplatelet therapy after acute coronary syndrome. N Engl J Med 2011;365:699-708.

12. Mehran R, Rao SV, Bhatt DL, et al. Standardized bleeding definitions for cardiovascular clinical trials: a consensus report from the bleeding academic research consortium. Circulation 2011;123:2736-47.

13. Mega JL, Braunwald E, Wiviott S, et al. Rivaroxaban in patients with a recent acute coronary syndrome. N Engl J Med 2012;366:9-19.
14. Granger CB, Alexander JH, McMurray JJV, et al. Apixaban versus warfarin in patients with atrial fibrillation. N Engl J Med 2011;365:981-92.

15. Patel MR, Mahaffey KW, Garg J, et al. Rivaroxaban versus warfarin in valvular atrial fibrillation. N Engl J Med 2010;376:975-83.

16. Wiviott SD, Braunwald E, McCabe $\mathrm{CH}$, et al. Prasugrel versus clopidogrel in patients with acute coronary syndromes. N Engl J Med 2007;357:2001-15.

17. Steg PG, James SK, Atar D, et al. ESC guidelines for the management of acute myocardial infarction in patients presenting with ST segment elevation. Eur Heart J 2012;33:2569-619.

18. Hamm CW, Bassand JP, Agewall S, et al. ESC guidleines for the management of acute coronary syndromes in patients presenting without persistent ST-segment elevation. Eur Heart J 2011;32:29993054 . 